\title{
KIF1B promotes glioma migration and invasion via cell surface localization of MT1-MMP
}

\author{
SONGYU CHEN ${ }^{1-4}$, MINGZHI HAN ${ }^{1,4}$, WEILIANG CHEN ${ }^{1,3,4}$, YING HE ${ }^{3,4}$, BIN HUANG $^{1,4}$, \\ PENG ZHAO $^{1,3,4}$, QIBING HUANG ${ }^{1,4}$, LIANG GAO $^{2}$, XUN QU $^{3}$ and XINGANG LI ${ }^{1,4}$ \\ ${ }^{1}$ Department of Neurosurgery, Qilu Hospital of Shandong University, Jinan, Shandong 250012; \\ ${ }^{2}$ Department of Neurosurgery, Shanghai Tenth People's Hospital, Tongji University, Shanghai 200072; \\ ${ }^{3}$ Institute of Basic Medical Sciences, Qilu Hospital of Shandong University, Jinan, Shandong 250012; \\ ${ }^{4}$ Brain Science Research Institute, Shandong University, Jinan, Shandong 250012, P.R. China
}

Received May 15, 2015; Accepted October 19, 2015

DOI: $10.3892 /$ or.2015.4426

\begin{abstract}
Malignant glioma is notorious for its aggressiveness and poor prognosis, and the invasiveness of glioma cells is the major obstacle. Accumulating evidence indicates that kinesin superfamily proteins (KIFs) may play key roles in tumor invasiveness, but the mechanisms remained unresolved. Our previous study demonstrated that membrane type 1-matrix metalloproteinase (MT1-MMP) was involved in Kinesin family member 1B (KIF1B)-modulated invasion of gastric cancer cells. Therefore, the role of KIF1B in glioma cell invasion and its relationship with MT1-MMP were explored in the present study. We found that aberrantly increased expression of KIF1B was associated with worse WHO pathological classification and Karnofsky performance status (KPS), which also showed a trend towards worse prognosis. In the Transwell assay, knockdown of KIF1B using siRNA repressed U87MG and A172 glioma cell migration and invasion. Silencing KIF1B inhibited expression of membranal MT1-MMP; however, the amount of MT1-MMP in the whole cell lysate was not affected. In conclusion, targeting KIF1B may be an option for anti-invasive therapies targeting glioma.
\end{abstract}

\section{Introduction}

Malignant glioma, especially glioblastoma multiforme (GBM), is one of the most aggressive forms of all human cancers, with a median survival time of 12-15 months for GBM and 2-5 years for anaplastic gliomas (1). Despite recent advances in therapeutic modalities, including surgery, chemotherapy and radiotherapy, the overall survival of GBM patients was

Correspondence to: Professor Xingang Li, Department of Neurosurgery, Qilu Hospital of Shandong University, 107 Wenhuaxi Road, Jinan, Shandong 250012, P.R. China

E-mail: lixg@sdu.edu.cn

Key words: glioma, KIF1B, pathology, neoplasm invasiveness, membrane type 1-matrix metalloproteinase not significantly improved over the past 20 years. Malignant gliomas display extensive infiltration of cells into the normal brain parenchyma, which makes complete surgical removal almost impossible. What makes the situation worse is that malignant glioma cells are highly resistant to radiotherapy and chemotherapy, thus, leading to recurrence (2). Furthermore, anti-angiogenic therapy using bevacizumab increased survival in patients with recurrent glioblastoma, it also increased tumor invasiveness (3). As is known, the invasiveness of glioma cells is the key problem in the management of malignant gliomas. To the best of our knowledge, there are no established antiinvasive therapies available.

Membrane type 1-matrix metalloproteinase (MT1-MMP, MMP-14), a key metalloproteinase with a C-terminal sequence that functions as membrane-anchoring domain, plays an important role during tumor invasion (4). Once transported to cell surface, MT1-MMP will be cleaved and activated. Activated MT1-MMP cleaves copious substrates, including another critical MMP, namely proMMP-2. Malignant gliomas overexpress MT1-MMP and upregulation of MT1-MMP in glioma cells correlates with their invasiveness $(5,6)$. Additionally, the expression of MT1-MMP negatively correlates with prognosis of glioma patients (7). The above results indicate that MT1-MMP is a key metalloproteinase in the process of glioma cell invasion.

KIFs are a family of molecular motors which drive the transport of certain cargoes such as protein complexes along microtubular tracks. They are involved in cellular functions, including cell division and intracellular transport. Accumulating evidence supports the important role of KIFs in tumor development and progression (8). The expression of kinesin family member $2 \mathrm{~A}$ (KIF2A) negatively correlated with prognosis of squamous cell carcinoma of the oral tongue (SCCOT) and breast cancer and knocking down KIF2A inhibited invasion $(9,10)$. Additionally, kinesin family member $3 \mathrm{~A}$ (KIF3A) correlated with clinicopathological factors of prostate cancer, while silencing KIF3A decreased proliferation and invasion (11). First discovered by Nangaku et al (12) in 1994, kinesin family member 1B (KIF1B) participated in not only axonal transport of synaptic vesicles and mitochondria, but also axon myelination and outgrowth $(13,14)$. We have 
reported that leptin stimulates MT1-MMP expression as well as its cell surface localization, which is dependent on KIF1B and consequently promoting invasion of gastric cancer cells (15). Neither the expression, nor the function of KIF1B in glioma has been reported, and its molecular mechanisms in tumorigenesis and progression require further investigation.

According to previous findings, we examined the protein and mRNA expression of KIF1B in glioma using tumor specimens as well as databases, and analyzed the correlation of KIF1B expression with pathological grades, Karnofsky performance status (KPS) and patient survival in this study. Furthermore, we identified that KIF1B promoted glioma cell migration and invasion and was involved in cell surface localization of MT1-MMP. These results suggest that increased KIF1B expression may facilitate the localization of MT1-MMP, thus promoting glioma cell invasion.

\section{Materials and methods}

Patients and specimens. The research was approved by the Ethics Committee of the Qilu Hospital. Archived paraffinembedded glioma tissues were collected from 67 patients who underwent surgery in the Department of Neurosurgery, Qilu Hospital of Shandong University (Shandong, China). The diagnosis of each case was confirmed by two pathologists according to the 2007 World Health Organization (WHO) classification (16). The tissue specimens included WHO I $(n=5)$ and WHO II $(n=13)$, WHO III $(n=13)$ and WHO IV $(n=28)$. We obtained written informed consent from all patients or their guardians.

Reagents and antibodies. The membrane protein extraction kit was purchased from Thermo Fisher Scientific (Rockford, IL, USA; cat no. 89826). KIF1B rabbit anti-human mAb for immunohistochemical analysis was from Santa Cruz Biotechnology (Santa Cruz, CA, USA; cat no. sc-28540). KIF1B and MT1-MMP rabbit anti-human $\mathrm{mAb}$ for western blot analysis were from Abcam (Cambridge, UK; cat no. ab69614 for KIF1B and ab51074 for MT1-MMP). $\beta$-actin rabbit anti-human $\mathrm{mAb}$ was from Cell Signaling Technology (Beverly, MA, USA; cat no. 4967).

Cell culture. The human glioma U87MG (referred to as U87 hereinafter for ease of presentation) cell line from the Culture Collection of the Chinese Academy of Sciences (Shanghai, China) was grown in Dulbecco's modified Eagle's medium (DMEM)/F12 medium containing 10\% fetal bovine serum (FBS) obtained from Thermo Fisher Scientific, 100 units $/ \mathrm{ml}$ penicillin, and $100 \mu \mathrm{g} / \mathrm{ml}$ streptomycin in humidified air with $5 \% \mathrm{CO}_{2}$ at $37^{\circ} \mathrm{C}$.

Immunohistochemical analysis. Paraffin-embedded tissues were deparaffinized and rehydrated. After antigen retrieval, all tissues were exposed to primary antibody (KIF1B, 1:200) overnight at $4^{\circ} \mathrm{C}$, and then incubated with poly-HRP (horseradish peroxidase) secondary antibodies (ZSGB-BIO, Beijing, China; cat no. SP-9001) for $30 \mathrm{~min}$ at $37^{\circ} \mathrm{C}$. Staining was observed with 3,3N-diaminobenzidine tertrahydrochloride and evaluated independently by two pathologists without prior knowledge of the clinicopathological information of the specimens. An immunohistochemical (IHC) score was generated as previously described, with slight modifications (17). Briefly, the IHC score was calculated by multiplying: i) the staining intensity (scored as: 0 , no staining; 1 , weak staining; 2 , moderate staining; and 3, strong staining); and ii) the percentage of KIF1B-positive glioma cells (scored as: 1, 1-10\%; 2, 11-50\%; 3 , $51-80 \%$; or $4,81-100 \%$ ). An IHC score of 9-12 was considered strong immunoreactivity $(+++), 5-8$ as moderate $(++), 1-4$ as weak $(+)$, and 0 was considered negative (-).

Quantitative reverse transcription-polymerase chain reaction ( $q R T-P C R)$. Total RNA was extracted using TRIzol reagent (Sangon Biotech Co., Ltd., Shanghai, China), treated with DNase I (Thermo Fisher Scientific) then reverse transcribed to cDNA using M-MuLV reverse transcriptase (Thermo Fisher Scientific), following the manufacturer's protocol. Quantitative polymerase chain reaction (PCR) was performed as previously described (18). PCR was performed using the following primers: GADPH forward, GGTGGTCTCCTCTGACTTC AACAG and reverse, GTTGCTGTAGCCAAATTCGTTGT; KIF1B forward, TGGCAGTTACTTCCTACACAGA and reverse, GGGAACGGCTACTTGTTTCAT.

Western blot analysis. Total protein from cells or tissues was extracted in RIPA buffer [50 mM Tris- $\mathrm{HCl}, \mathrm{pH} 7.5,1 \%$ IGEPAL CA-630 (v/v), $150 \mathrm{mM} \mathrm{NaCl}$, and $0.5 \%$ sodium deoxycholate] and quantified using the BCA method. Equal amounts of proteins from each sample were separated on $10 \%$ sodium dodecyl sulfate-polyacrylamide gel electrophoresis and transferred onto nitrocellulose membranes. The membranes were blocked for $1 \mathrm{~h}$ at room temperature with TBST $(50 \mathrm{mM}$ Tris-HCl, $150 \mathrm{mM} \mathrm{NaCl}, 0.05 \%$ Tween-20, pH 7.0) containing $5 \%$ non-fat dry milk and then incubated with the primary antibody [KIF1B (1:200), MT1-MMP (1:1,000), calnexin $(1: 1,000)$ and $\beta$-actin $(1: 1,000)]$ overnight at $4^{\circ} \mathrm{C}$. The membranes were exposed to the horseradish peroxidase-labeled secondary antibodies $(1: 2,000)$ for $1 \mathrm{~h}$ at room temperature and then were detected by enhanced chemiluminescence detection system (Amersham Life Science Inc., Pittsburgh, PA, USA).

RNA interference. Small interference RNA (siRNA) constructs targeting KIF1B, and stable negative control were designed and purchased from Shanghai GenePharma Co., Ltd. (Shanghai, China). For transient silencing, 3x $10^{5}$ cells/well were seeded onto 6 -well plates and transfected with relevant siRNA (100 nmol/well) using Lipofectamine RNAiMAX reagent (Invitrogen, Carlsbad, CA, USA; cat no. 13778150), following the manufacturer's protocol.

Cell invasion and migration assay. For Transwell Matrigel invasion assay, $5 \times 10^{4}$ cells in $100 \mu 1$ of serum-free medium were plated onto the upper chamber of 24-well Transwell inserts ( $8 \mu \mathrm{m}$ pores; BD Biosciences, Bedford, MA, USA) coated with Matrigel, as previously described with slight modifications (19). The lower chamber was filled with $600 \mu \mathrm{l}$ medium containing 20\% FBS. After 36-48 h, the non-invaded cells were gently scraped off by cotton swab. The migrated cells were fixed by $10 \%$ formalin, stained with crystal violet and counted. Five random fields of each well were photographed and cell numbers were determined by Kodak MI software. 
As for Transwell migration assay, $2 \times 10^{4}$ cells in $100 \mu \mathrm{l}$ of serum-free medium were plated onto the upper chamber of the same inserts and migrated for $10 \mathrm{~h}$. The other steps were the same as described above.

Immunofluorescence staining. After KIF1B siRNA transfection, U87 cells were harvested and then plated on glass slides in 24-well culture plates at a concentration of $1 \times 10^{5}$ cells/well for $12 \mathrm{~h}$. Thereafter, the cells were fixed with a $4 \%$ formaldehyde solution in PBS, permeabilized with $0.5 \%$ Triton X-100 in PBS, stained for filamentous actin using Alexa-Fluor 488-labeled phalloidin dyes (Cell Signaling Technology; cat no. 8878) according to the manufacturer's instructions. The cells were counterstained with 4',6-diamidino-2-phenylindole (DAPI) and examined under an Olympus BX61 fluorescence microscope.

Cell viability assay. The cell viability assay was previously described (20), with slight modifications. Briefly, $2.5 \times 10^{3}$ cells in $200 \mu \mathrm{l}$ medium per well were seeded into a 96 -well plate. At the time indicated, $20 \mu \mathrm{l}$ MTT $(5 \mathrm{mg} / \mathrm{ml})$ was added into each well. The medium was incubated for another $4 \mathrm{~h}$ in the dark. Consequently, the medium was expirated. A total of $200 \mu \mathrm{l}$ DMSO was used to dissolve the formazan grain. The absorbance at $570 \mathrm{~nm}$ was measured using an Infinite 200 PRO microplate reader (Tecan Schweiz AG, Männedorf, Switzerland).

In silico analysis. The Oncomine database and Repository of Molecular Brain Neoplasia Data (REMBRANDT) of the National Cancer Institute (http://www.betastasis.com/) were used to analyze mRNA expression of KIF1B and its prognostic value in glioma.

Statistical analysis. The SPSS software package (version 17.0; SPSS, Inc., Chicago, IL, USA) was used for all statistical analysis. Chi-square test and Fisher's exact test were applied to analyze the correlation of clinicopathologcial parameters and KIF1B expression. Other data from experiments were analyzed by paired Student's t-test or one-way ANOVA analysis of variance wherever appropriate.

\section{Results}

The expression of KIF1B correlates with the clinicopathologcial information of glioma. First, we investigated the expression of KIF1B mRNA in 39 glioma tissue samples using qRT-PCR. The levels of KIF1B mRNA increased with the ascending pathological classification of glioma $(\mathrm{P}<0.05$; Fig. 1A). The aberrant expression of KIF1B was further validated by immunohistochemical analysis of 67 glioma tissue samples (Fig. 1B). The glioma tissuse samples were grouped as KIF1B ${ }^{\text {Low }}$ (IHC score: - to + ) and as KIF1B ${ }^{\text {High }}$ (IHC score: ++ to +++$)$. As shown in Table I, KIF1B expression was significantly associated with glioma grade according to the WHO classification and KPS.

The aberrant expression and prognostic value of KIF1B is validated in the databases. To further investigate KIF1B expression and its clinical significance in gliomas, we used
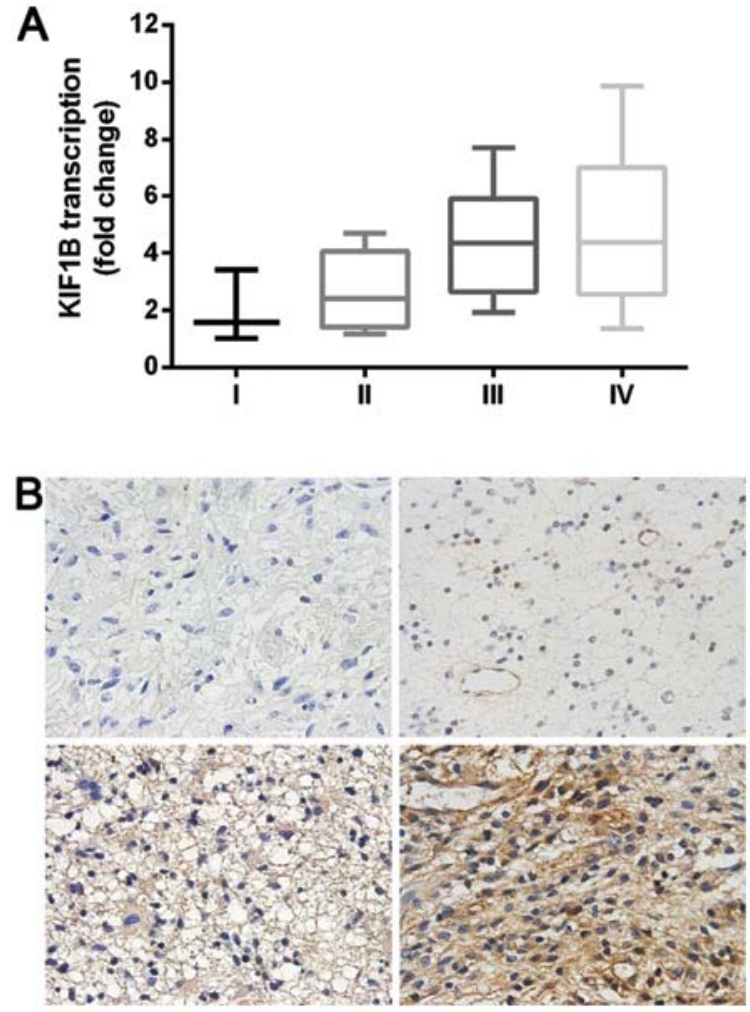

Figure 1. The expression of KIF1B in glioma tissue samples. (A) The transcriptional levels of KIF1B in 39 glioma tissue samples. Samples 1-3 were WHO I; samples 4-11 were WHO II; samples 12-24 were WHO III; samples 13-39 were WHO IV. The expression is normalized to the lowest expression (sample 2). The relationship between KIF1B mRNA expression and pathological classification of glioma was determined by one-way ANOVA analysis; $\mathrm{P}<0.05$. Error bars represent mean \pm SD. (B) Representative microphotographs of KIF1B immunostaining in glioma tissue samples (magnification, x400). They were choroid plexus papilloma (top left: WHO I, -), fibrillary astrocytoma (top right: WHO II, +), anaplastic astrocytoma (lower left: WHO III, ++ to +++) and glioblastoma (lower right: WHO IV,+++ ).

Table I. The relationship between KIF1B immunohistochemical expression and clinicopathologcial parameters of patients.

\begin{tabular}{lcccc}
\hline Variables & Total & $\begin{array}{c}\text { IHC score } \\
(-/+)\end{array}$ & $\begin{array}{c}\text { IHC score } \\
(++/+++)\end{array}$ & P-value \\
\hline Gender & & & & \\
$\quad$ Male & 42 & 27 & 15 & 0.5155 \\
Female & 25 & 18 & 7 & \\
Age (years) & & & & \\
$\geq 50$ & 44 & 32 & 12 & 0.1799 \\
$<50$ & 23 & 13 & 10 & \\
KPS & & & 10 & 0.0254 \\
$>70$ & 43 & 33 & 12 & \\
$\leq 70$ & 24 & 12 & & \\
WHO grade & & & 18 & 0.0180 \\
High (III-IV) & 41 & 23 & 4 & \\
Low (I-II) & 26 & 22 & & \\
\hline
\end{tabular}

IHC, immunohistochemical; KPS, Karnofsky performance status; WHO, World Health Organization. P-values were determined by Chi-square test and Fisher's exact test. 

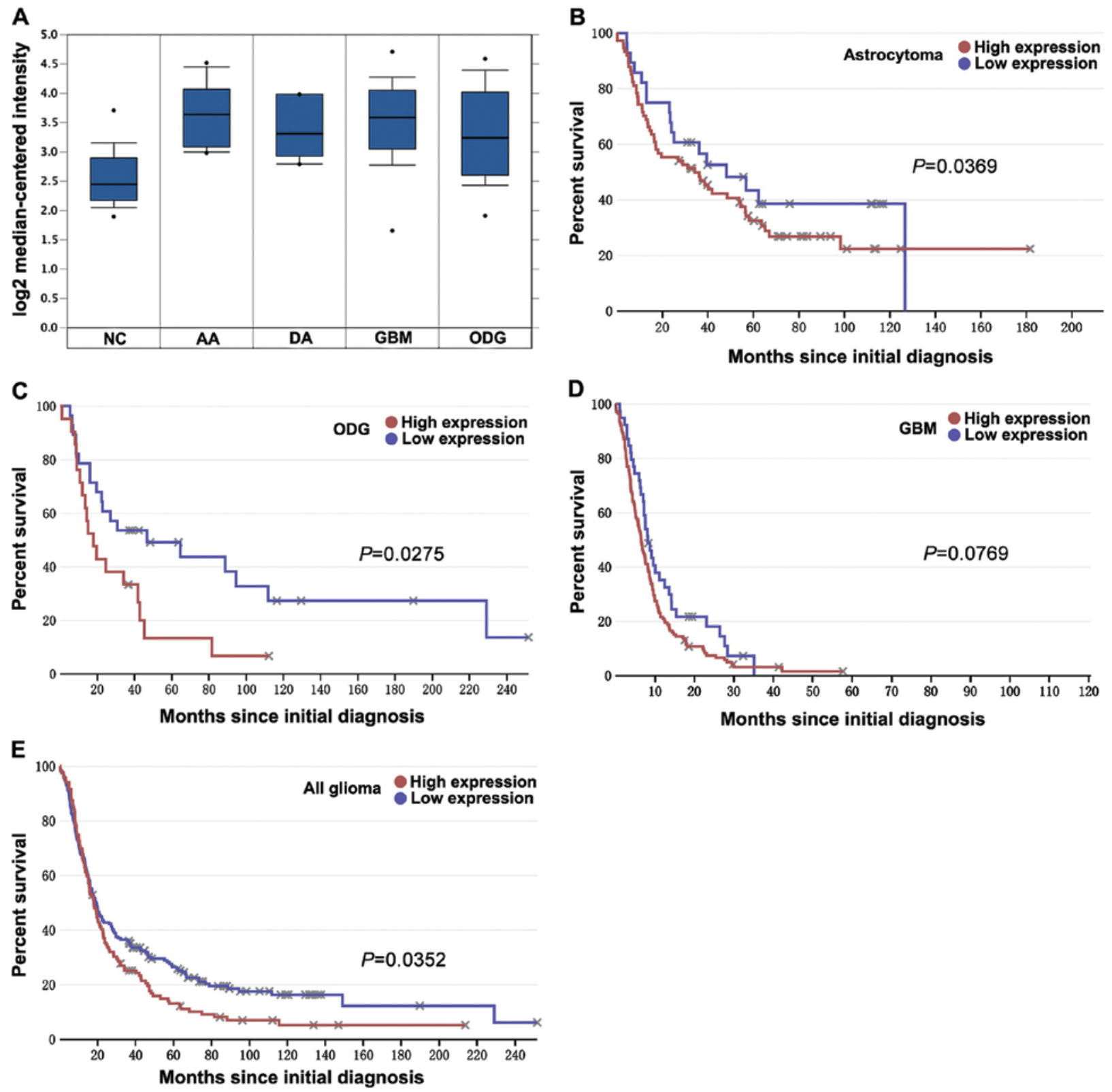

Figure 2. Expression and prognostic value of KIF1B in gliomas of the databases. (A) Oncomine microarray data analysis for KIF1B expression in different gliomas vs. normal brain tissue is shown. The Student's t-tests were conducted using the Oncomine software; compared with normal brain, each type of glioma has a P-value $<0.001$. NC represents non-tumor controls $(n=23)$, AA represents anaplastic astrocytoma (grade III, $n=19$ ), DA represents diffuse astrocytoma (grade II, $n=7$ ), GBM represents glioblastoma (grade IV, n=81) and ODG represents oligodendroglioma (grade II, $n=50$ ). The boxes represent the 25th through 75th percentiles; the horizontal lines represent the medians; the points represent the end of the ranges. (B-E) According to different KIF1B mRNA expression for overall survival of glioma patients, Kaplan-Meier curves were plotted (using data extracted from the REMBRANDT database at http://www.betastasis.com/). Briefly, (B) atrocytoma, (C) oligodendroglioma (ODG), (D) glioblastoma (GBM) and (E) all gliomas. P-values were obtained from log-rank tests.

two databases to examine the differential expression and prognostic value of KIF1B in glioma. Analysis based on a set of Oncomine data showed that KIF1B mRNA expression was markedly upregulated in anaplastic astrocytoma $(n=19)$, diffuse astrocytoma $(n=7)$, GBM (grade $n=81)$ and oligodendroglioma (grade $n=50)$ than in non-tumor controls $(n=23$; each $\mathrm{P}<0.001$; Fig. 2A). According to the data extracted from REMBRANDT, patients with high KIF1B mRNA-expressing astrocytoma ( $\mathrm{P}=0.0369$; Fig. $2 \mathrm{~B})$ and oligodendroglioma $(\mathrm{P}=0.0275$; Fig. $2 \mathrm{C})$ showed poorer prognosis compared to patients with low KIF1B mRNA-expressing ones. Although there is only a tendency towards poorer prognosis in GBM
( $P=0.0769$; Fig. 2D), a significant prognostic difference still remains in all glioma $(\mathrm{P}=0.0352$; Fig. $2 \mathrm{E})$.

Silencing KIF1B inhibits invasion and migration of glioma cells, not affecting cell viability. KIF1B was aberrantly expressed in glioma tissue samples and correlated with pathological classification and KPS of patients. It was revealed that the pathological classification of glioma is related to invasiveness (21). Moreover, we previously proved that KIF1B promoted invasiveness of gastric cancer cells (15). Hence, KIF1B was antagonized by siRNA in vitro in U87 as well as A172 glioma cell lines. The silencing efficacy of selected 


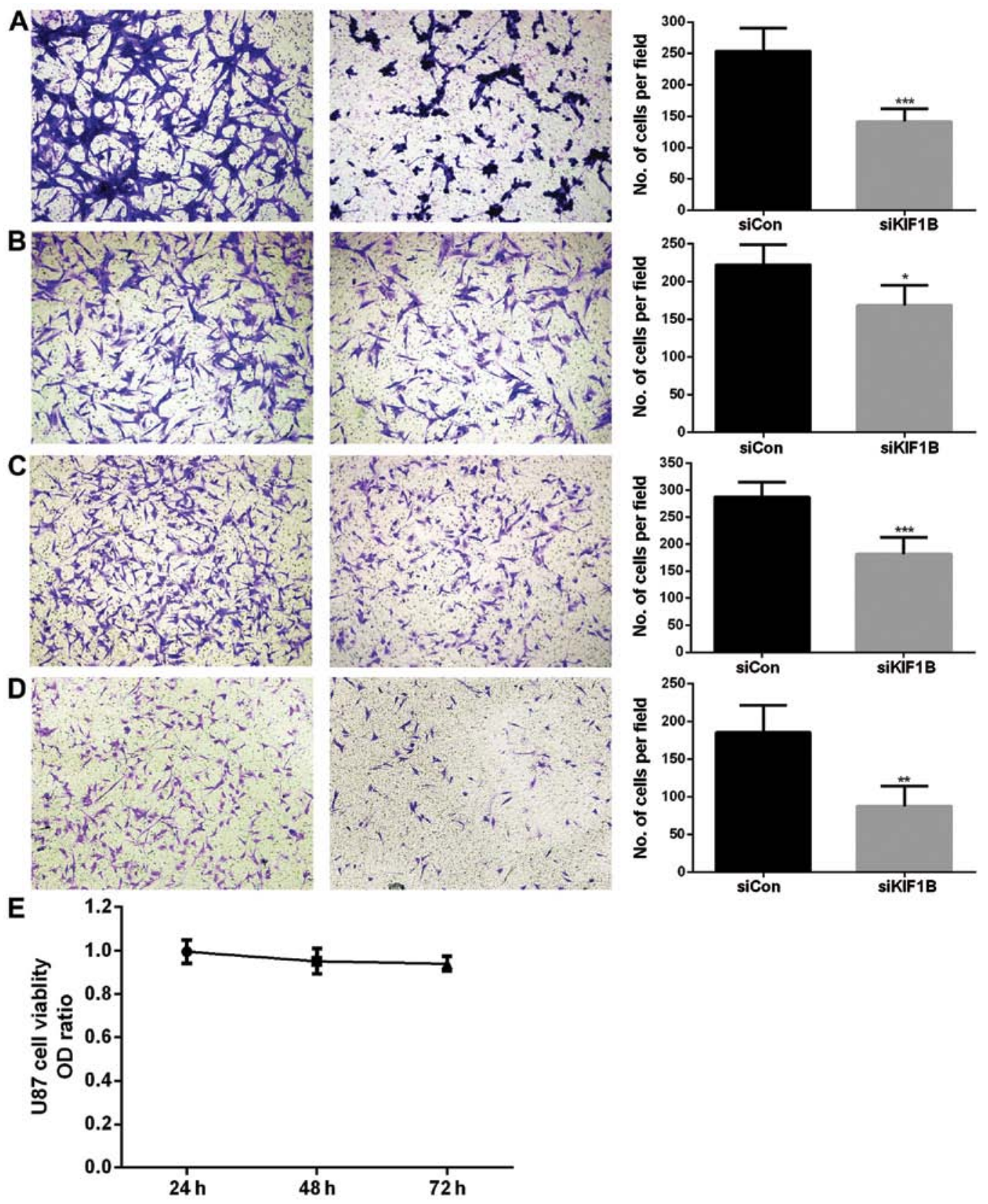

Figure 3. The effect of knocking down KIF1B on glioma cell invasion and migration. In Transwell assays, the numbers of (A) invading U87 and (C) A172 cells were markedly lower in the KIF1B-siRNA treating group. Consistent with Matrigel invasion assays, the numbers of (B) migrating U87 and (D) A172 cells were significantly lower in the KIF1B-siRNA treating group. (E) U87 cell viability of KIF1B-siRNA treating group was not affected at indicated time-points. OD ratio indicates absorbance at $570 \mathrm{~nm}$ of KIF1B-siRNA treating group compared to that of control-siRNA treating group. ${ }^{* * * *} \mathrm{P}<0.001,{ }^{* * *} \mathrm{P}<0.01,{ }^{*} \mathrm{P}<0.05, \mathrm{n}=5$. P-value was calculated by Student's t-test. Error bars represent mean \pm SD

KIF1B siRNA was verified by qRT-PCR (data not shown). Compared with control siRNA treatment, knocking down KIF1B significantly inhibited both invasion $(\mathrm{P}<0.001$; Fig. 3A) and migration $(\mathrm{P}<0.05$; Fig. $3 \mathrm{~B})$ of U87 glioma cells. Silencing KIF1B in A172 cells also remarkably inhibited their invasiveness $(\mathrm{P}<0.001$; Fig. 3C) and migratory $(\mathrm{P}<0.01$; Fig. 3D) ability. KIF1B-siRNA was transfected into U87 glioma cells and did not affect cell viability after 24,48 and $72 \mathrm{~h}(\mathrm{P}=0.25$; Fig. 3E). Knocking down KIF1B in A172 glioma cells showed similar results (data not shown).

Silencing KIF1B inhibits glioma invasion and migration through downregulation of membranal MT1-MMP.
MT1-MMP is transported to cell surface in order to exert its biological function (4). Our previous co-immunoprecipitation data have demonstrated that MT1-MMP and KIF1B interacts with each other (15). Considering the important role of KIF1B on intracellular transport and the effects of MT1-MMP on tumor invasion, we speculated that KIF1B may affect the cell localization of MT1-MMP, and then influence cell migration and invasion in glioma. After knocking down KIF1B ( $\mathrm{P}<0.001$; Fig. 4A and B), the protein levels of MT1-MMP in the whole U87 glioma cell lysate were not affected $(\mathrm{P}=0.485 ;$ Fig. $4 \mathrm{~A}$ and $\mathrm{C})$. However, the expression of membranal MT1-MMP was remarkably lower in the KIF1BsiRNA treating group $(\mathrm{P}<0.01$; Fig. $4 \mathrm{~A}$ and $\mathrm{C})$. Additionally, 

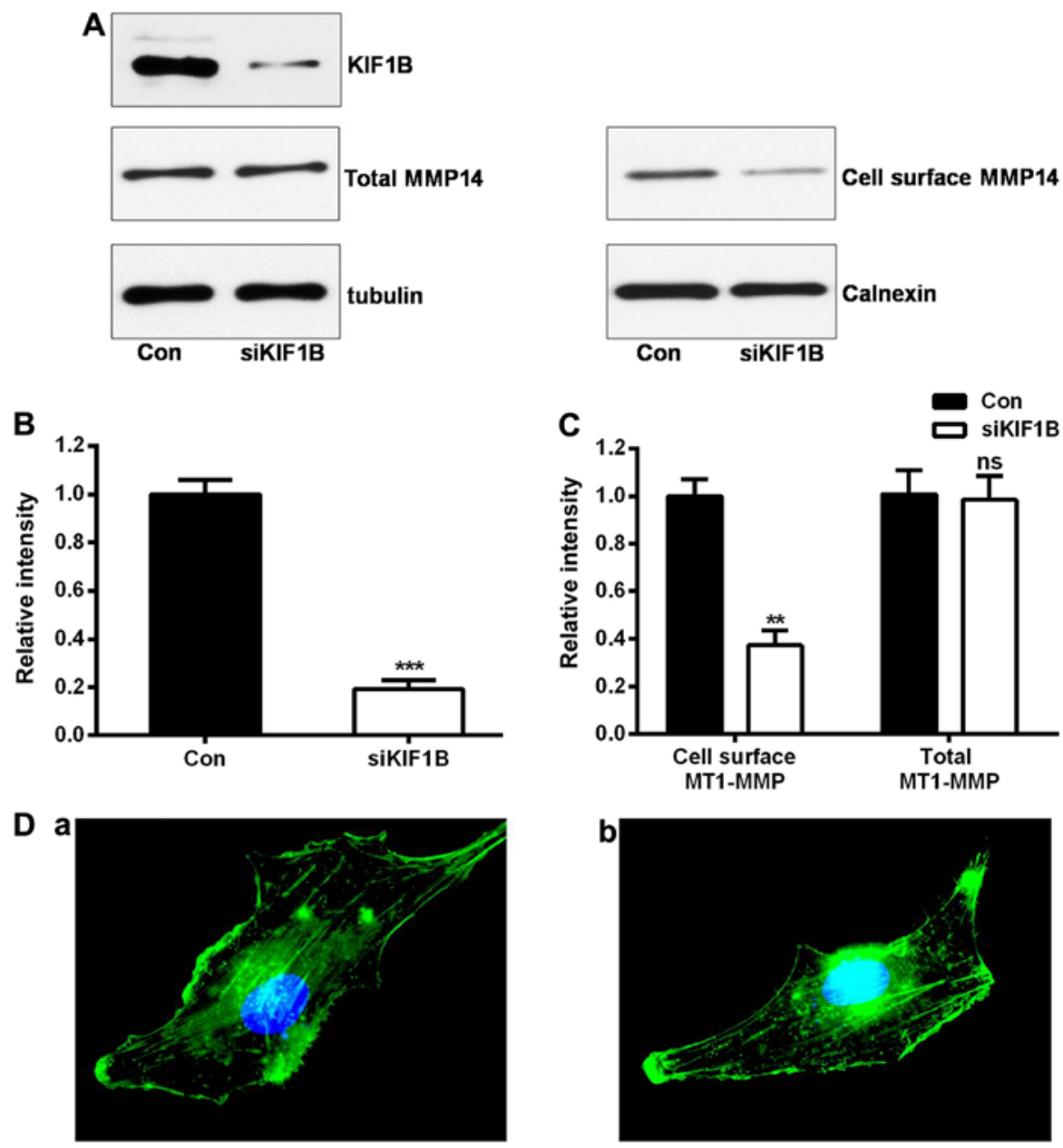

Figure 4. Silencing KIF1B decreases the expression of membranal MT1-MMP and does not affect cytoskeleton of glioma cells. (A-C) Twenty-four hours after siRNA transfection, the protein levels of KIF1B and MT1-MMP in whole U87 glioma cell lysate were determined by western blot analysis. The protein levels of membranal MT1-MMP in U87 glioma cells were also analyzed. ${ }^{* * *} \mathrm{P}<0.001,{ }^{* *} \mathrm{P}<0.01$; ns indicates not statistically significant, $\mathrm{n}=3$. $\mathrm{P}$-values were calculated by Student's t-test. Error bars represent mean \pm SD. (D) Filamentous actin of U87 glioma cells were visualized by Alexa-Fluor 488 -labeled phalloidin staining at the same time-point. Representative microphotographs are shown (magnidication, x600): (a) A U87 glioma cell treated with control siRNA, and (b) treated with KIF1B-siRNA.

knocking down KIF1B did not affect the cytoskeleton of U87 glioma cells (Fig. 4D). In conclusion, KIF1B promoted glioma cell invasion via intracellular transport of MT1-MMP.

\section{Discussion}

In the present study, we revealed that malignant gliomas express high levels of KIF1B both at the levels of mRNA and protein. Aberrant expression of KIF1B correlated with the WHO pathological classification and KPS. Unfortunately, we failed to follow up a number of included patients. Then we accessed the REMBRANDT database and discovered that glioma aberrantly expressing KIF1B correlated with poorer prognosis. Moreover, we verified that gliomas have increased expression of KIF1B compared to non-tumor tissue samples. Previous studies have revealed that KIF2C (22) and KIF14 (23) are aberrantly expressed in gliomas and they are independent markers for prognosis. KIF23 was aberrantly expressed in glioma tissue samples, and knockdown of KIF23 suppressed glioma cell proliferation (24). These KIFs are crucial for the regulation of cell cycle and mitosis, which are emerging targets for human cancer control. However, the known function of KIF1B is intracellular transportation.

Accumulating evidence shows that specific members of the 23 known human matrix metalloproteinases (MMPs), especially MT1-MMP, alter tumor cell behavior and stimulate cancer progression. In terms of glioma, expression of MT1-MMP both correlates with and increases with histological grade of malignancy (25). Moreover, it is known that MT1-MMP is vital in glioma cell malignant behavior including growth, invasion, migration and angiogenesis (25). Besides, MT1-MMP may be a key regulator of $\mathrm{CD} 133^{+}$ glioma stem cells (26). Previously, our group demonstrated that leptin promotes MT1-MMP expression as well as its cell surface localization in a KIF1B-dependent manner in gastric cancer (15). According to the above evidence, we presumed that MT1-MMP is also the key regulator in KIF1B-mediated glioma cell invasion and migration.

Malignant gliomas are notorious for their invasiveness. In the Transwell assay, knocking down KIF1B via siRNA inhibited invasion and migration of U87 and A172 glioma cells. The possibility that KIF1B may influence cell viability 
was excluded through MTT assay. Similarly, silencing KIF1B did not affect the overall expression of MT1-MMP protein in U87 glioma cells. As a crucial metalloproteinase in tumor invasion, MT1-MMP is regulated at various levels, including intracellular trafficking. It is known that the localization on the cell surface is required for protease activity of MT1-MMP and activation of subsequent MMPs. Then we examined the membranal MT1-MMP level, and our results showed the membranal MT1-MMP was downregulated in U87 glioma cells. On the other hand, siRNA-mediated KIF1B knockdown did not obviously change the cytoskeleton of glioma cells. Therefore, our results suggested that the membranal MT1-MMP was the key point in KIF1B-mediated glioma cell invasion.

Recent clinical as well as basic evidence indicates that glioma's escape from antiangiogenic therapies (e.g. bevacizumab) at least in part correlates with augmented invasion (27). What is more, glioma overexpresses MT1-MMP in order to enhance angiogenesis via upregulation of VEGF (28). Based on our findings, anti-invasive therapies targeting KIF1B is an option in treating glioma, particularly in combination with antiangiogenic therapies. For instance, siRNA therapy is believed to have the potential to effectively reduce expression of cancer-specific genes (29,30). Knockdown of KIF1B by siRNA may suppress glioma progression. Alternatively, specific inhibitors have been developed to downregulate other KIFs (8). The same strategy is another choice in the case of KIF1B.

In conclusion, we discovered gliomas overexpressed KIF1B, which correlated with worse prognosis. Moreover, aberrant expression of KIF1B was associated to both pathological grade and KPS. To the best of our knowledge, we showed for the first time that KIFs were involved in glioma invasion. KIF1B played a key role in glioma invasiveness via membranal MT1-MMP. Our data indicated that KIF1B may be a promising target for glioma.

\section{References}

1. Wen PY and Kesari S: Malignant gliomas in adults. N Engl J Med 359: 492-507, 2008.

2. Furnari FB, Fenton T, Bachoo RM, Mukasa A, Stommel JM, Stegh A, Hahn WC, Ligon KL, Louis DN, Brennan C, et al: Malignant astrocytic glioma: Genetics, biology, and paths to treatment. Genes Dev 21: 2683-2710, 2007.

3. Lucio-Eterovic AK, Piao Y and de Groot JF: Mediators of glioblastoma resistance and invasion during antivascular endothelial growth factor therapy. Clin Cancer Res 15: 4589-4599, 2009.

4. Poincloux R, Lizárraga F and Chavrier P: Matrix invasion by tumour cells: A focus on MT1-MMP trafficking to invadopodia. J Cell Sci 122: 3015-3024, 2009.

5. Fillmore HL, VanMeter TE and Broaddus WC: Membrane-type matrix metalloproteinases (MT-MMPs): Expression and function during glioma invasion. J Neurooncol 53: 187-202, 2001.

6. Guo P, Imanishi Y, Cackowski FC, Jarzynka MJ, Tao HQ, Nishikawa R, Hirose T, Hu B and Cheng SY: Up-regulation of angiopoietin-2, matrix metalloprotease-2, membrane type 1 metalloprotease, and laminin 5 gamma 2 correlates with the invasiveness of human glioma. Am J Pathol 166: 877-890, 2005.

7. Wang L, Yuan J, Tu Y, Mao X, He S, Fu G, Zong J and Zhang Y Co-expression of MMP-14 and MMP-19 predicts poor survival in human glioma. Clin Transl Oncol 15: 139-145, 2013.

8. Rath $\mathrm{O}$ and Kozielski F: Kinesins and cancer. Nat Rev Cancer 12 $527-539,2012$

9. Wang CQ, Qu X, Zhang XY, Zhou CJ, Liu GX, Dong ZQ, Wei FC and Sun SZ: Overexpression of Kif2a promotes the progression and metastasis of squamous cell carcinoma of the oral tongue. Oral Oncol 46: 65-69, 2010.
10. Wang J, Ma S, Ma R, Qu X, Liu W, Lv C, Zhao S and Gong Y: KIF2A silencing inhibits the proliferation and migration of breast cancer cells and correlates with unfavorable prognosis in in breast cancer. BMC Cancer 14: 461, 2014.

11. Liu Z, Rebowe RE, Wang Z, Li Y, Wang Z, DePaolo JS, Guo J, Qian C and Liu W: KIF3a promotes proliferation and invasion via Wnt signaling in advanced prostate cancer. Mol Cancer Res 12: 491-503, 2014.

12. Nangaku M, Sato-Yoshitake R, Okada Y, Noda Y, Takemura R, Yamazaki H and Hirokawa N: KIF1B, a novel microtubule plus end-directed monomeric motor protein for transport of mitochondria. Cell 79: 1209-1220, 1994.

13. Lyons DA, Naylor SG, Scholze A and Talbot WS: Kif1b is essential for mRNA localization in oligodendrocytes and development of myelinated axons. Nat Genet 41: 854-858, 2009.

14. Zhao C, Takita J, Tanaka Y, Setou M, Nakagawa T, Takeda S, Yang HW, Terada S, Nakata T, Takei Y, et al: Charcot-MarieTooth disease type $2 \mathrm{~A}$ caused by mutation in a microtubule motor KIF1Bbeta. Cell 105: 587-597, 2001.

15. Dong Z, Xu X, Du L, Yang Y, Cheng H, Zhang X, Li Z, Wang L, Li J, Liu H, et al: Leptin-mediated regulation of MT1-MMP localization is KIF1B dependent and enhances gastric cancer cell invasion. Carcinogenesis 34: 974-983, 2013.

16. Louis DN, Ohgaki H, Wiestler OD, Cavenee WK, Burger PC, Jouvet A, Scheithauer BW and Kleihues P: The 2007 WHO classification of tumours of the central nervous system. Acta Neuropathol 114: 97-109, 2007.

17. Yang YM, Feng AL, Zhou CJ, Liang XH, Mao HT, Deng BP, Yan S, Sun JT, Du LT, Liu J, et al: Aberrant expression of chemokine receptor CCR4 in human gastric cancer contributes to tumor-induced immunosuppression. Cancer Sci 102: 1264-1271, 2011

18. Zhao P, Li XG, Yang M, Shao Q, Wang D, Liu S, Song H, Song B, Zhang Y and Qu X: Hypoxia suppresses the production of MMP-9 by human monocyte-derived dendritic cells and requires activation of adenosine receptor A2b via cAMP/PKA signaling pathway. Mol Immunol 45: 2187-2195, 2008.

19. Wang W, Sima N, Kong D, Luo A, Gao Q, Liao S, Li W, Han L, Wang J, Wang S, et al: Selective targeting of HPV-16 E6/E7 in cervical cancer cells with a potent oncolytic adenovirus and its enhanced effect with radiotherapy in vitro and vivo. Cancer Lett 291: 67-75, 2010 .

20. Liu Q, Li G, Li R, Shen J, He Q, Deng L, Zhang C and Zhang J: IL-6 promotion of glioblastoma cell invasion and angiogenesis in U251 and T98G cell lines. J Neurooncol 100: 165-176, 2010.

21. Palfi S, Swanson KR, De Boüard S, Chrétien F, Oliveira R, Gherardi RK, Kros JM, Peschanski M and Christov C: Correlation of in vitro infiltration with glioma histological type in organotypic brain slices. Br J Cancer 91: 745-752, 2004.

22. Bie L, Zhao G, Wang YP and Zhang B: Kinesin family member 2C (KIF2C/MCAK) is a novel marker for prognosis in human gliomas. Clin Neurol Neurosurg 114: 356-360, 2012

23. Wang Q, Wang L, Li D, Deng J, Zhao Z, He S, Zhang Y and Tu Y: Kinesin family member 14 is a candidate prognostic marker for outcome of glioma patients. Cancer Epidemiol 37: 79-84, 2013.

24. Takahashi S, Fusaki N, Ohta S, Iwahori Y, Iizuka Y, Inagawa K, Kawakami Y, Yoshida K and Toda M: Downregulation of KIF23 suppresses glioma proliferation. J Neurooncol 106: 519-529, 2012.

25. Ulasov I, Yi R, Guo D, Sarvaiya P and Cobbs C: The emerging role of MMP14 in brain tumorigenesis and future therapeutics. Biochim Biophys Acta 1846: 113-120, 2014

26. Annabi B, Laflamme C, Sina A, Lachambre MP and Béliveau R: A MT1-MMP/NF-kappaB signaling axis as a checkpoint controller of COX-2 expression in CD $133^{+}$U87 glioblastoma cells. J Neuroinflammation 6: 8, 2009.

27. Hardee ME and Zagzag D: Mechanisms of glioma-associated neovascularization. Am J Pathol 181: 1126-1141, 2012.

28. Deryugina EI, Soroceanu L and Strongin AY: Up-regulation of vascular endothelial growth factor by membrane-type 1 matrix metalloproteinase stimulates human glioma xenograft growth and angiogenesis. Cancer Res 62: 580-588, 2002.

29. Akita H, Kogure K, Moriguchi R, Nakamura Y, Higashi T, Nakamura T, Serada S, Fujimoto M, Naka T, Futaki S, et al: Nanoparticles for ex vivo siRNA delivery to dendritic cells for cancer vaccines: programmed endosomal escape and dissociation. J Control Release 143: 311-317, 2010.

30. Yagi N, Manabe I, Tottori T, Ishihara A, Ogata F, Kim JH, Nishimura S, Fujiu K, Oishi Y, Itaka K, et al: A nanoparticle system specifically designed to deliver short interfering RNA inhibits tumor growth in vivo. Cancer Res 69: 6531-6538, 2009. 